\title{
Moderne Senologie aus Sicht von Prävention und Früherkennung
}

\author{
I. Schreer, Ehrenpräsidentin der Deutschen Gesellschaft für Senologie
}

Prävention meint im Kontext des Mammakarzinoms sekundäre Prävention. Denn bisher stehen keine Substanzen oder Maßnahmen im Sinne einer primären Prävention zur Verfügung, die in einem akzeptablen Nutzen-Risikoverhältnis gesunden Frauen mit nicht erhöhtem Brustkrebs-Risiko angeboten werden könnten zur Verhinderung der Erkrankung.

Brustkrebs-Früherkennung versteht sich als übergeordneter Begriff für alle Strategien, die es erlauben, Brustkrebs in einem Stadium zu erkennen, in dem das Fortschreiten des malignen Prozesses gestoppt werden kann. Ziel ist die Senkung der Inzidenz im Fall der Entdeckung eines Vorstadiums und vor allem eine Verringerung der Sterblichkeit an der Erkrankung. Aber auch die Chance, weniger eingreifend behandeln zu müssen als Folge der Reduktion fortgeschrittener Tumorstadien, hat hohen Wert bezüglich der Lebensqualität der Betroffenen.

Wir unterscheiden populationsbezogene (Screening) und individuelle Früherkennungsstrategien.

Zweifelsfrei hat das Mammografiescreening, d.h. der Einsatz eines einzigen Tests (der Mammografie) zur Sekundärprävention, seine Effektivität unter Beweis gestellt sowohl innerhalb großer internationaler randomisiert kontrollierter Studien als auch außerhalb, im Rahmen der Normalversorgung ( Schweden und die Niederlande). Keine andere Methode konnte bisher sekundäre Prävention mit so günstigem Schaden-Nutzen-Verhältnis erreichen. Vorraussetzung ist ein stringentes Qualitätssicherungskonzept, wie es in den Europäischen Empfehlungen zur Qualitätssicherung des Mammografiescreenings und der Diagnostik (4. Auflage) vorgegeben ist.

Betrachtet man sekundäre Prävention unter einem populationsbezogenen Aspekt, müssen hohe Detektionsraten, günstige Stadienverteilung und Mortalitätsreduktion auch unter Kosten-Nutzen-Aspekten gesehen werden. Ungute Extreme einer Betrachtung in Hinblick auf die Kosten sind z. B. die 3-Jahres-Intervalle im englischen Mammografiescreening, die der Wachstumsgeschwindigkeit nur des hoch differenzierten, indolenten Mammakarzinoms gerecht werden sowie die ehemals extrem restriktiv vorgegebenen Abklärungsraten von 1\% im niederländischen Screening. Der Preis waren hohe Intervall-Karzinomraten von bis zu $50 \%$.

Andererseits bedarf es aber auch der sorgfältigen Balance zwischen hohen Detektionsraten und Überdiagnose/Übertherapie. Die stetige, umfassende wissenschaftliche Evaluation bevölkerungsbezogener Projekte ist daher zwingend, um gegebenenfalls frühzeitig steuernd eingreifen oder verbessern zu können.

Die Vorraussetzungen für ein umfassendes Früherkennungskonzept wurden mit der ersten Aktualisierung der Stufe-3-Leitlinie Brustkrebs-Früherkennung in Deutschland erarbeitet unter $\mathrm{Zu}-$ grundelegung der vorhandenen Evidenz, konsensbasiert und niedergelegt unter der Maßgabe, dieses Wissen letztendlich den Frauen verfügbar zu machen mit dem Ziel verbesserter Versorgung. Senologische Aufgabe der Brustkrebs-Früherkennung ist es, jeder Frau die Chance der Sekundär-Prävention zu eröffnen und nicht nur einem begrenzten Alterssegment. Das entsprechende Leitlinien-Statement lautet: „Brustkrebs-Früherkennungsuntersuchungen sind für alle Frauen nützlich, deren individuelles Risiko-Profil durch Alter und/oder weitere Risikofaktoren (endogene und exogene hormonelle Faktoren, Brustdrüsendichte, reproduktives Verhalten, Lebensstil, erbliche Faktoren) beschrieben ist (LOE 2 a, Empfehlungsgrad A)“. Leider ist bis heute die Implementierung der Leitlinien-Inhalte in die Versorgung nur rudimentär und punktuell vonstatten gegangen. Größte Hindernisse sind berufspolitische, fachgruppenspezifische und versorgungsstrukturbedingte Interessen und Widerstände.

Ein Früherkennungskonzept muß sich am individuellen Risiko orientieren. Größtes populationsbezogenes Risiko ist das Alter. Daher haben Frauen inzwischen seit 2010 jenseits der Mammografie-Screening-Altersgrenze von 70Jahren im Rahmen der sogenannten kurativen Versorgung Anspruch auf eine Früherkennungs-Mammografie. Diese Indikation muss natürlich gegenüber altersbedingter begleitender Morbidität abgewogen werden.

Die Datenlage für Frauen unter 50Jahren hat sich weiter verbessert, einerseits durch die randomisiert-kontrollierte Studie aus Großbritannien („Age Trial“) als auch durch aktuelle Publikationen aus den Niederlanden und Schweden. Die publizierten Ergebnisse weisen auf eine gegenüber dem üblichen Alterssegment vergleichbare Mortalitätsreduktion hin in einer Größenordnung zwischen $19 \%$ für das Age-Trial (immer noch mit zu kurzer Nachbeobachtungszeit und daher knapp unter Signifikanz-Niveau) und 26\% im schwedischen Mammografiescreening junger Frauen (SCRY) für die eingeladenen Frauen bzw. 29\% für die Teilnehmerinnen. Die Ergebnisse einer Fall-Kontroll-Studie unter Einbeziehung junger Frauen aus dem Nijmegen-Screening kommt zu einer statistisch signifikanten Mortalitätsreduktion von $50 \%$. Es besteht somit kein Grund mehr, Frauen unter 50Jahren aus dem Mammografiescreening auszuschließen.

Es ist ebenfalls nicht einzusehen, ein qualitätssicherndes Konstrukt nur für das Screening anzubieten. Die vergleichbare Umsetzung von Prozess- und Ergebnisqualität leitlinienkonform für die sogenannte kurative Versorgung in Schleswig-Holstein führte zu einem statistisch signifikanten Vorteil für das Gesamtüberleben der Teilnehmerinnen mit einer HR von 0,78 (95\% CI 0,65-0,94). Die Zukunft muss es daher sein, die Altersgrenzen im Mammografiescreening zu öffnen, die neutrale wissenschaftliche Evaluation auf die Beine zu stellen, Frauen intensiv zu informieren über Nutzen und Risiken und die so genannte kurative Versorgung in eine qualitätssichernde Struktur einzubinden, um so z.B. auch die Sonografie bei ACR3/4 gezielt anbieten zu können. Weitergehendes Zukunftsziel sollte eine individualisierte Präventionsstrategie sein auf der Basis zu entwickelnder „Marker“.

\section{Bibliografie}

Dol http://dx.doi.org/10.1055/s-0031-1271492

Senologie 2011; 8: 71

(c) Georg Thieme Verlag KG Stuttgart · New York · ISSN 1611-6453

\section{Korrespondenzadresse}

Prof. Dr. med. Ingrid Schreer

Karlstr. 2

22085 Hamburg

ingrid.schreer@hamburg.de 